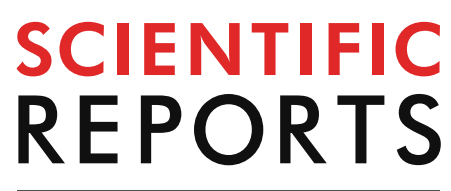

natureresearch

Check for updates

\title{
OPEN Aligned nanofiber scaffolds improve functionality of cardiomyocytes differentiated from human induced pluripotent stem cell-derived cardiac progenitor cells
}

Mei Ding ${ }^{1,5 \bowtie}$, Henrik Andersson ${ }^{1,5}$, Sofia Martinsson ${ }^{2}$, Alan Sabirsh ${ }^{3}$, Anna Jonebring ${ }^{1}$, Qing-Dong Wang ${ }^{2}$, Alleyn T. Plowright ${ }^{4}$ \& Lauren Drowley ${ }^{2}$

Cardiac progenitor cells (CPCs), capable of differentiating into multiple cardiac cell types including cardiomyocytes (CMs), endothelial cells, and smooth muscle cells, are promising candidates for cardiac repair/regeneration. In vitro model systems where cells are grown in a more in vivo-like environment, such as $3 \mathrm{D}$ cultures, have been shown to be more predictive than $2 \mathrm{D}$ culture for studying cell biology and disease pathophysiology. In this report, we focused on using Wnt inhibitors to study the differentiation of human iPSC-CPCs under 2D or 3D culture conditions by measuring marker protein and gene expression as well as intracellular $\mathrm{Ca}^{2+}$ oscillation. Our results show that the 3D culture with aligned nanofiber scaffolds, mimicing the architecture of the extracellular matrix of the heart, improve the differentiation of iPSC-CPCs to functional cardiomyocytes induced by Wnt inhibition, as shown with increased number of cardiac Troponin T (cTnT)-positive cells and synchronized intracellular $\mathrm{Ca}^{2+}$ oscillation. In addition, we studied if 3D nanofiber culture can be used as an in vitro model for compound screening by testing a number of other differentiation factors including a ALK5 inhibitor and inhibitors of BMP signaling. This work highlights the importance of using a more relevant in vitro model and measuring not only the expression of marker proteins but also the functional readout in a screen in order to identify the best compounds and to investigate the resulting biology.

Heart failure after myocardial infarction is a clinical condition that causes high morbidity and mortality ${ }^{1}$. The only currently available curative treatment of end-stage heart failure is heart transplantation, whereas other treatment options merely slow the progression of the disease. Therefore, there is a large unmet medical need for treating heart failure that is driven by the loss of functional cardiomyocytes (CMs) which occurs during myocardial infarction ${ }^{2}$. Thus, cardiac regeneration is now seen as an attractive strategy for repairing damaged heart tissue and for treating heart failure ${ }^{2-4}$. Recent studies have demonstrated that transplantation of human embryonic stem cell (ESC)- or induced pluripotent stem cell (iPSC)-derived cardiac progenitor cells (CPCs), $\mathrm{CMs}$, or cardiac muscle patches, formed new functional myocardium and improved cardiac function in rodents, swine, non-human primates, and in humans ${ }^{5-9}$. Therefore, CPCs are very promising candidates for improving

\footnotetext{
${ }^{1}$ Discovery Biology, Discovery Sciences, R\&D, AstraZeneca, Gothenburg, Sweden. ${ }^{2}$ Bioscience Cardiovascular, Research and Early Development, Cardiovascular, Renal and Metabolism (CVRM), BioPharmaceuticals R\&D, AstraZeneca, Gothenburg, Sweden. ${ }^{3}$ Advanced Drug Delivery, Pharmaceutical Sciences, R\&D, AstraZeneca, Gothenburg, Sweden. ${ }^{4}$ Medicinal Chemistry, Research and Early Development, Cardiovascular, Renal and Metabolism (CVRM), BioPharmaceuticals R\&D, AstraZeneca, Gothenburg, Sweden. ${ }^{5}$ These authors contributed equally: Mei Ding and Henrik Andersson. ${ }^{\circledR}$ email: mei.ding@astrazeneca.com
} 
the function of dysfunctional myocardium, and for the identification of molecules increasing differentiation of CPCs to CMs which could enhance the therapeutic value of these cells for heart failure.

CPCs can be generated from iPSCs and we have previously demonstrated the multi-lineage differentiation capacity of iPSC-derived CPCs into different cardiac lineages: CMs, smooth muscle cells (SMCs) and endothelial cells $(\mathrm{ECs})^{10}$. Screening of a diverse compound library using human iPSC-CPCs showed that XAV939 (an inhibitor of Wnt pathway signaling), Dorsomorphin (DM) (an inhibitor of AMP-activated kinase (AMPK) and BMP signaling), RepSox (an inhibitor of TGF-b type 1 receptor ALK5) or other structurally diverse inhibitors of ALK $5^{10}$ promote cardiac differentiation of human iPSC-CPCs. This study demonstrated that the human iPSC-CPC assay can be run in a medium to high-throughput phenotypic screening approach to identify novel and potent regulators of cardiac regeneration and potentially yield new drugs or new therapeutic mechanisms for treating heart failure.

Our previous iPSC-CPC differentiation study was performed in standard two-dimensional (2D) culture. It is well known that cells cultured on 2D flat glass or plastic planar surfaces often show different morphology and function compared to cells in the native tissue ${ }^{11}$. Many examples across different cell types have shown that cell morphology, function, and fate are influenced by the physical interactions of cells with the extracellular matrix $(\mathrm{ECM})^{12-16}$. During the past 20 years, increased efforts have been made to culture cells in a more in vivo-like environment by using three-dimensional (3D) culture systems with relevant matrix components. Numerous studies have demonstrated that in vitro cellular models with cells grown in $3 \mathrm{D}$ culture, which structurally mimic the architecture of the ECM of the native tissue, have higher predictivity in in vitro models than 2D culture models for studying cell biology and disease pathophysiology, and for identifying therapeutic agents ${ }^{17-19}$. For example, HepG2 liver cancer cells in 3D spheroids produce their own ECM and are highly organized and 'tissue-like"20, fibroblasts cultured with collagen gels and fibronectin-containing matrices that mimic in vivo 3D environment exert different drug responsiveness compared to cells growing in $2 \mathrm{D}$ cultures ${ }^{21}$. However, using $3 \mathrm{D}$ culture models, such as spheroids, organoids and organ-on-a-chip in drug discovery for screening large numbers of compounds (e.g. in a phenotypic screening campaign) can still be very challenging as these more complex assays are difficult to adapt to plate-based medium-to-high throughput screening and automation. In recent years, a number of plate-based 3D culture models, such as low attachment and hanging drop plates for spheroids, plates with nanofibrous scaffolds composed of electrospun synthetic nanofibers, and plates coated with hydrogels, have become commercially available. Such 3D cultures have been investigated in different cellular models to improve physiological relevance, including human adipose-derived stem cells ${ }^{15}$, rat hippocampal embryonic neurons $s^{22}$, bovine pulmonary artery smooth muscle cells ${ }^{23}$, and hepatocytes ${ }^{24,25}$. Adult CMs in vivo have an elongated morphology, but, CMs cultured in standard 2D condition do not show elongated morphology. Numerous studies have shown that aligned nanofiber scaffolds guide CM cell alignment along the direction of fiber orientation, promote adaptation of an elongated CM morphology, and improve CM function and maturation when CMs are cultured in 3D aligned nanofiber scaffolds ${ }^{26-32}$. The potential of engineered cardiac tissue like constructs obtained by seeding CMs on aligned nanofibers or into a 3D fibrin scaffold for treating myocardial infarction has been demonstrated ${ }^{8,32}$. In addition, several groups also reported that nanofiber scaffolds enhance cardiac differentiation of stem cells or progenitor cells ${ }^{33-37}$. Thus, there is a clear rationale to investigate if nanofiber scaffolds can improve iPCS-CPCs differentiation into CMs to provide a more effective and relevant model or phenotypic assay, particularly if scaleable for medium-to-high throughput drug discovery.

For this study, 384-well 3D nanofiber plates with aligned polycaprolactone (PCL) nanofiber scaffolds, which structurally mimic the architecture of CMs in the heart ${ }^{38}$, was chosen to investigate the effect of $3 \mathrm{D}$ scaffolds on human iPSC-CPC cardiac differentiation triggered by Wnt inhibition that is reported to promote iPSC-CPC cardiac differentiation. Protein and gene expression of CM and SMC markers, and intracellular $\mathrm{Ca}^{2+}$ oscillation were used for cardiac differentiation assessment. In addition, we studied if 3D nanofiber culture can be used as an in vitro model for compound screening by testing alternative molecules which have been shown to differentiate iPSC-CPCs in 2D culture.

\section{Results}

Wnt signaling inhibition induced differentiation of human iPSC-CPCs in 3D and 2D culture. Human iPSC-CPCs were treated with $10 \mu \mathrm{M}$ XAV939, $1.1 \mu \mathrm{M} 53 \mathrm{AH}$ (a structurally diverse inhibitor of Wnt signalling), or DMSO control in triplicates. Cells were fixed at day 7 or day 14 of differentiation, then stained for cardiac Troponin T (cTnT) and smooth muscle actin (aSMA) for studying iPSC-CPC differentiation. These concentrations and time points were selected based on our previously obtained knowledge for the differentiation of CPCs to CMs and that described in the literature ${ }^{10,39}$. Figure 1 shows an outline of the human iPSC-CPC differentiation protocol, and double immunostaining with cTnT and aSMA of cells at day 14 in 3D versus 2D culture treated with XAV939, 53AH or DMSO, as well as aligned fibers on 3D nanofiber plates.

To quantify protein expression levels, automated analysis was performed using Columbus image analysis software (Perkin Elmer). Nuclear and cytoplasmic regions were segmented using images of Hoechst and combined images from cTnT and aSMA, respectively. cTnT and aSMA intensities were calculated, and cells were classified as cTnT-positive and/or aSMA-positive. At day 7 of differentiation, 53AH treatment resulted in a significantly higher fraction of cTnT-positive cells and higher cTnT intensity in 3D nanofiber culture compared to the 2D cultures (Fig. 2a,b). As the differentiation time increased from 7 to 14 days, the percentage and cTnT intensity of cTnT-positive cells in 53AH and XAV939 treated cells in 3D culture were increased (Fig. 2f,g).

The percentage of aSMA-positive cells was higher in 2D culture treated with 53AH or XAV939 at day 7 (Fig. 2c), as well as in 2D culture treated with 53AH or DMSO at day 14, compared to the corresponding 3D cultures (Fig. 2h). Higher aSMA intensity was also observed in aSMA-positive cells in 3D culture treated with 53AH and XAV939 at day 14 (Fig. 2i). No significant differences were found for other treatments at day 7 or 14 
a

Plate cells in 2D or 3D plates Compound dose

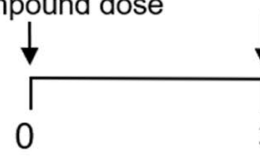

Fix \& stain cells with 7 days treatment

fresh

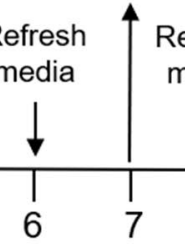

Days

Fix \& stain cells with 14 days treatment

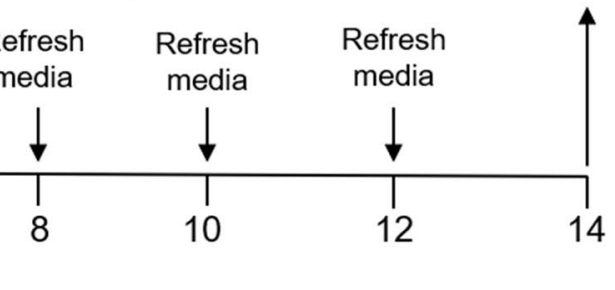

b aSMA cTnT Hoechst / cTnT / aSMA

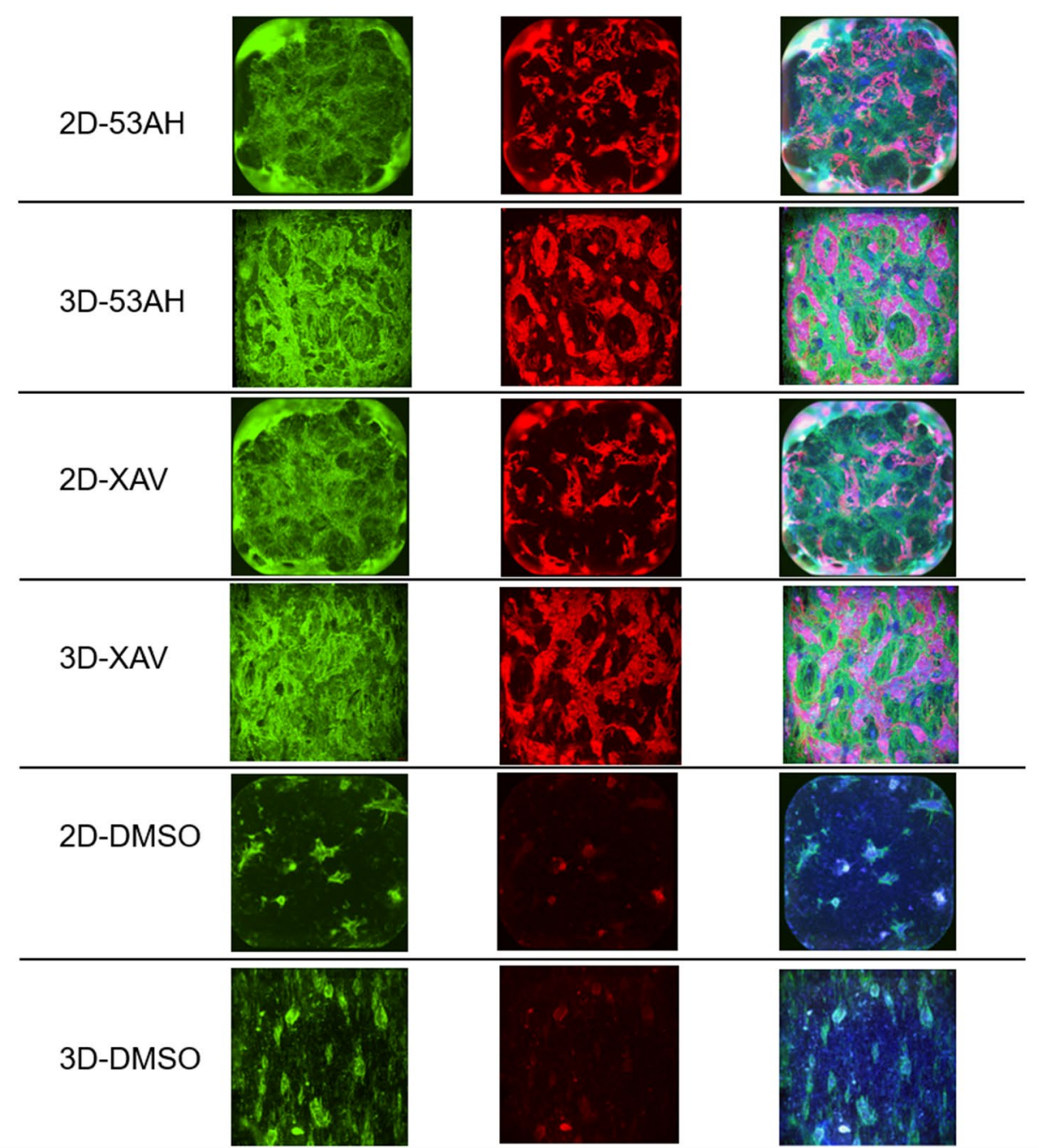

C

Aligned fibers on nanofiber plate

Figure 1. Schematic outline of the CPC differentiation protocol and immunofluorescence staining of CPC differentiation in 3D aligned nanofiber plates and 2D plates. (a) Outline of the human iPSC-CPC differentiation protocol: Human iPSC-CPCs were plated in $3 \mathrm{D}$ aligned nanofiber or $2 \mathrm{D}$ plates with the addition of compounds and DMSO control from day 0 to day 3 , followed by culture in assay medium until day 7 or day 14 of differentiation. (b) Representative images of cells treated with $1.1 \mu \mathrm{M} 53 \mathrm{AH}, 10 \mu \mathrm{M}$ XAV939, or DMSO control at day 14 of differentiation indicate that human iPSC-CPCs are competent for differentiation to cardiomyocytes (express cardiomyocyte marker cTnT) and cells expressing aSMA. (c) A phase contrast image of aligned nanofiber plate. These images were acquired at $\times 4$ magnification. 


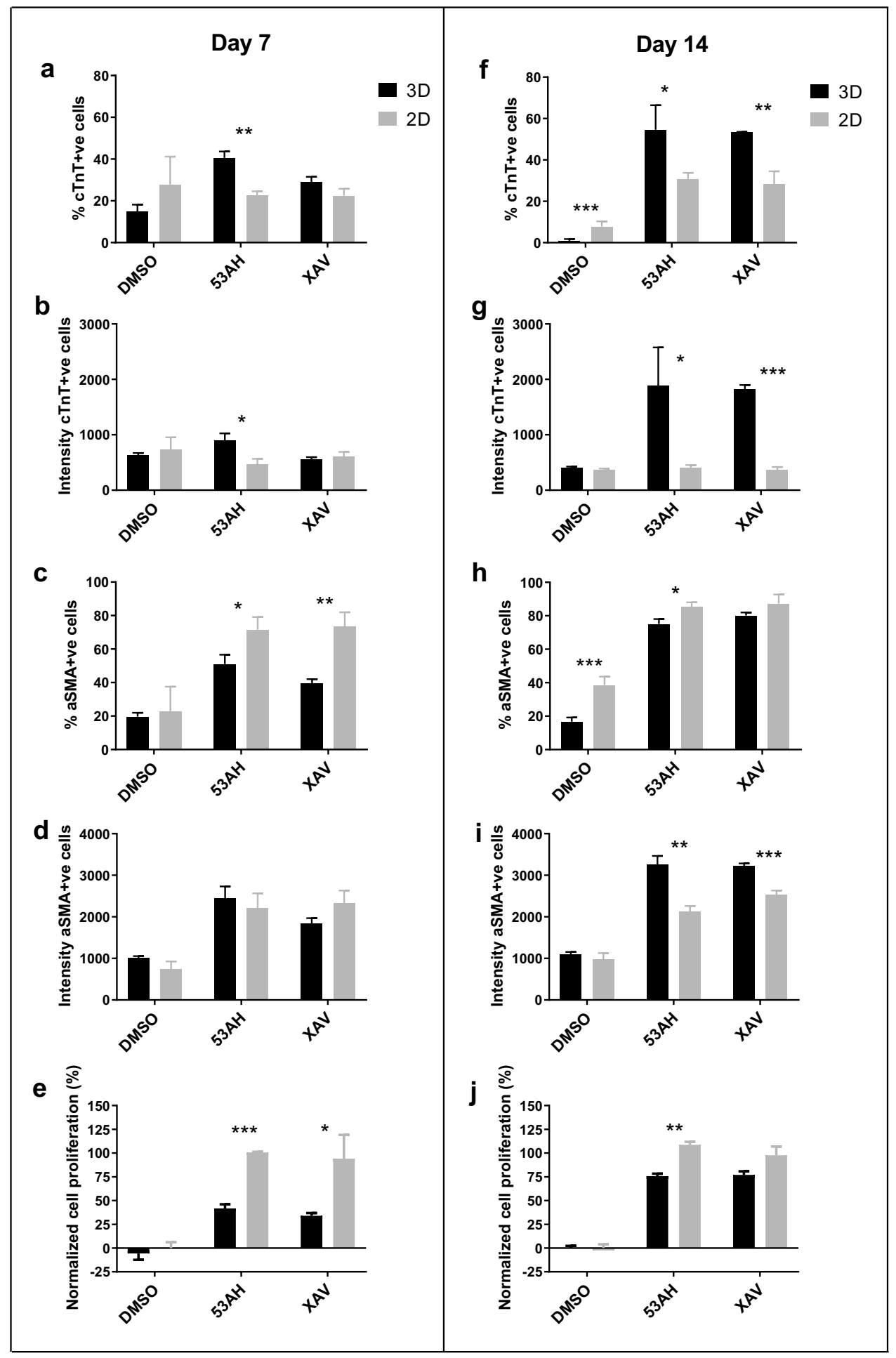

Figure 2. Effect of Wnt signaling inhibition on CPC differentiation and cell proliferation in $3 \mathrm{D}$ vs $2 \mathrm{D}$ culture. Human iPSC-CPCs were plated in 3D aligned nanofiber and 2D plates with the addition of DMSO control, $1.1 \mu \mathrm{M} 53 \mathrm{AH}, 10 \mu \mathrm{M}$ XAV939 from day 0 to day 3, followed by culture in assay medium until day 7 or day 14 of differentiation. The expression of cTnT and $\alpha$ SMA were studied by acquiring images at $\times 20$ magnification. (a-e) At day 7 of differentiation in 2D and 3D cultures, \% cTnT-positive cells, cTnT intensity of cTnT-positive cells, $\%$ of aSMA-positive cells, aSMA intensity of aSMA-positive cells, and effect on cell proliferation in 2D and 3D cultures. $(\mathbf{f}-\mathbf{j})$ At day 14 of differentiation, data of $\%$ cTnT-positive cells, cTnT intensity of cTnT-positive cells, $\%$ of aSMA-positive cells, aSMA intensity of aSMA-positive cells, and effect on cell proliferation. The effect on cell proliferation was normalized as the percentage of cell proliferation based on the on-plate DMSO controls. Results are presented as mean \pm SEM. $n=3 .{ }^{*} \mathrm{p}<0.05,{ }^{* *} \mathrm{p}<0.01,{ }^{* *} \mathrm{p}<0.001$ indicate significant differences between $3 \mathrm{D}$ and $2 \mathrm{D}$ cultures. 
between 3D and 2D culture. Treatment with 53AH and XAV939 also increased aSMA intensity in 3D culture as the differentiation time increased from 7 to 14 days (Fig. 2d,i).

Our results also show that 53AH and XAV939 treatments induced significant cell proliferation in 2D culture at day 7 compared to the DMSO treatment (Fig. 2e). As the differentiation time increased to day 14 (Fig. 2j), the cell numbers were maintained at a similar level as seen at day 7 , indicating that cell proliferation occurred mainly during the period of day 0 to day 7 , and cells did not proliferate significantly between day 7 and 14 . However, in 3D nanofiber plates, compound treatments induced less cell proliferation with only $34-42 \%$ proliferation at day 7 and $76 \%$ proliferation at day 14 , as compared to around $100 \%$ cell proliferation in $2 \mathrm{D}$ culture at day 7 and 14. These results indicate that cells proliferate less in 3D nanofiber culture compared with standard 2D culture.

Adult CMs in the heart have an elongated morphology. Studies from several groups have shown that aligned nanofiber scaffolds produced in their labs guide CM alignment along the fiber orientation and promote adaptation of an elongated CM morphology ${ }^{26-28}$. Nanofiber scaffolds in the 384-well aligned nanofiber plates from Nanofiber Solutions are relatively thin with a thickness of $\sim 20 \mu \mathrm{m}$. Our nanofiber plate validation results show that human iPSC-CMs seeded directly on 3D aligned nanofiber plates and cultured for 10 days had an elongated morphology resembling native CMs in the heart, which is consistent with the data reported previously although the nanofiber scaffolds are thinner than those others have used (Fig. S1 in Supplementary Information). Conversely, CMs seeded in 2D culture did not elongate or orientate in any particular direction. Interestingly, CMs differentiated from iPSC-CPCs on 3D aligned nanofibers did not show elongated morphology as shown in Fig. 1. To better understand morphology of cells differentiated from iPSC-CPCs, the 3D and 2D plates with cells from day 14 of differentiation were imaged confocally at $3 \mathrm{z}$-planes using $20 \times$ objective at $6 \mu \mathrm{m}$ intervals. The confocal images show that many aSMA-positive cells located closer to the fibers in XAV939 treated 3D culture tended to display elongated nuclei aligned to the orientation of the nanofibers (plane 1 in panel $b$ and f/Fig. 3), while cTnT-positive cells did not show a clear alignment with the fibers (plane 1, 2, 3 in panel d/Fig. 3). Cells in 2D culture plates did not show nuclei or cell morphology alignment (plane a, c, e in Fig. 3). The analysis of Width to Length ratio (WtL) shows that nucleus WtL ratio for cells treated with XAV939 in 2D culture were $0.54 \pm 0.01$ for plane $1,0.55 \pm 0.01$ for plane 2, $0.56 \pm 0.01$ for plane 3, respectively, indicating no significant WtL ratio difference between planes in 2D culture. While nucleus WtL ratio for cells treated with $10 \mu \mathrm{M}$ XAV939 in 3D culture were $0.47 \pm 0.03$ for plane $1,0.54 \pm 0.02$ for plane $2,0.58 \pm 0.01$ for plane 3 . The lower WtL ratio at plane 1 in $3 \mathrm{D}$ suggests that nuclei at plane 1 in $3 \mathrm{D}$ culture were more elongated when comparing with nuclei upwards in the same sample, or comparing with nuclei on the same plane in 2D culture. Several reports have shown that nuclear morphology can be used as a surrogate for cell morphology ${ }^{40,41}$. The observed nuclear elongation aligned to the orientation of the nanofibers suggests a cell morphology alignment. 53AH treatment in 3D induced a similar effect on nuclear morphology as the cells treated with XAV939 (Fig. S2 in Supplementary Information).

To further assess the effect of 3D nanofiber scaffolds on human iPSC-CPCs differentiation, cardiac and smooth muscle cell genes were analyzed. The RT-PCR results show that there is a higher expression of cardiac marker gene MYH7 in 53AH and XAV939 treated cells in 3D nanofiber plates compared with 2D plates at day 14 of differentiation. 53AH treatment also resulted in a higher expression level of TNNT2 in 3D nanofiber plates. However, 53AH and XAV939 treated cells show similar levels of SCN5A, MYH6, and KCNJ4 gene expression in $2 \mathrm{D}$ and 3D culture although these compound treated cells showed higher levels of SCN5A and MYH6 gene expression compared to DMSO vehicle treated cells. A slightly higher expression of GJA1 was observed in 3D culture compared to 2D culture. Moreover, a high expression of ACTA2 gene was observed in 53AH or XAV939 treated cells in 3D culture (Fig. 4).

Contractile activity is one characteristic of functional CMs. Intracellular $\mathrm{Ca}^{2+}$ has been shown to be a surrogate for assessing CM contraction. In this study, treated cells were inspected under a microscope every day from day 5 post-compound treatment. Cells treated with XAV939 or 53AH in 3D nanofiber culture began displaying spontaneous contraction at day 6-7. At day 14, XAV939 and 53AH treated cells showed large beating areas in $3 \mathrm{D}$ culture, while only small beating clusters were observed in $2 \mathrm{D}$ culture. To further investigate the effect of 3D nanofiber scaffolds on CM function, we studied intracellular $\mathrm{Ca}^{2+}$ oscillation of differentiated cells in 3D and 2D culture using FLIPR Calcium $5 \mathrm{Ca}^{2+}$ sensitive dye. 53AH or XAV939 treated cells in 3D nanofiber plates showed spontaneous synchronized $\mathrm{Ca}^{2+}$ oscillation at day 7 and 14 . In comparison, no clear $\mathrm{Ca}^{2+}$ oscillation was observed in $2 \mathrm{D}$ culture of cells treated with $53 \mathrm{AH}$ or XAV939 at day 7 or 14 . Representative intracellular $\mathrm{Ca}^{2+}$ recordings of cells treated with 53AH, XAV939 or DMSO in 3D versus 2D at day 7 and 14 are shown in Fig. 5a-d. CPC differentiated CMs in 3D nanofiber culture retained adrenergic responsiveness, as shown with increased $\mathrm{Ca}^{2+}$ oscillation rate induced by the addition of isoproterenol (Fig. $5 \mathrm{c}, \mathrm{d}$ ), which is confirmed by the quantification data showing an increased $\mathrm{Ca}^{2+}$ oscillation peak frequency at the isoproterenol stimulation condition as compared to the basal condition (Fig. 5e). The basal peak frequency in 53AH treated iPSC-CPCs in 3D nanofiber culture at day 14 was 20.1 beats per minute (BPM), which was slightly lower than the iPSC-CMs basal peak frequency, 24.2 BPM, with iPSC-CMs seeded directly on 3D aligned nanofiber plates and maintained for 10 days (Fig. S1). In addition, both 53AH and XAV939 treated cells in 3D showed a small increase in average peak amplitude (although not significant) was observed as the differentiation time increased from day 7 to 14 (Fig. 5f).

Effect of BMP signaling inhibition and ALK5 inhibitor on human iPSC-CPC differentiation in 3D and 2D culture. To further understand if 3D nanofiber culture in general is a better in vitro model for screening cardiac differentiation of human iPSC-CPCs, we tested DM and DMH1 (BMP signaling inhibitors) and RepSox (ALK5 inhibitor) that were identified to promote human iPSC-CPC cardiac differentiation using $2 \mathrm{D}$ culture $^{10}$. DMH1 and RepSox treatments resulted in both a high percentage and high cTnT intensity of cTnTpositive cells in $3 \mathrm{D}$ culture compared to the $2 \mathrm{D}$ culture only at day 14 . DM induced a higher percentage and high cTnT intensity of cTnT-positive cells in 3D at day 7, but the not at day 14 although there is still a similar 


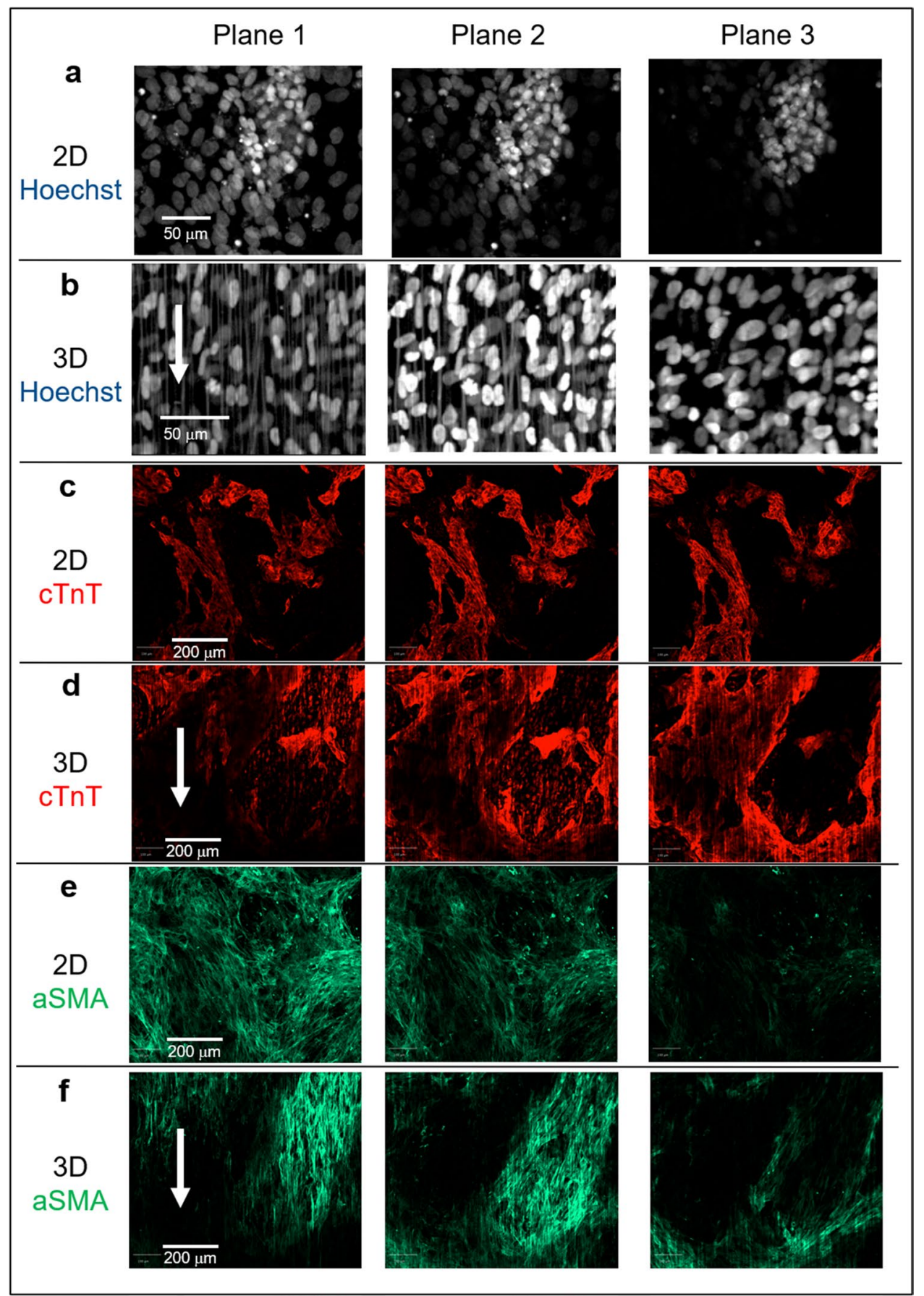

Figure 3. Confocal microscopy of CPC differentiation treated with XAV939 in 3D vs 2D culture. Human iPSC-CPCs were plated in 3D aligned nanofiber and 2D plates with the addition of $10 \mu \mathrm{M}$ XAV939 from day 0 to day 3 , followed by culture in assay medium until day 14 of differentiation. Hoechst staining (gray color), and expression of cTnT (red color) and aSMA (green color) were studied by acquiring images at $3 \mathrm{z}$-planes using $\times 20$ air objective at $6 \mu \mathrm{m}$ intervals. Plane 1 is close to the plate bottom level, Plane 2 is $6 \mu \mathrm{m}$ above plane 1 , and Plane 3 is $12 \mu \mathrm{m}$ above plane 1. (a-f) Representative images of cells treated with $10 \mu \mathrm{M}$ XAV939. Cells located closer to the fibers in 3D aligned nanofiber culture displayed elongated nuclei aligned to the orientation of the nanofibers (plane 1 in panel b). cTnT-positive CMs (panel d) were found to be located relatively distant from the fibers compared with aSMA-positive cells (panel f). Arrows have been added to plane 1 panel (b), (d) and (f) to highlight the fibers orientation.

trend (Fig. 6a,b,h,i). The percentage of aSMA-positive cells in 2D was higher than in 3D culture treated with DM at day 7 and 14 (Fig. 6c,j). aSMA-positive cells also showed higher aSMA intensity in 3D culture treated with DMH1 and RepSox at day 7 and 14. A high cell proliferation rate was observed in DM and DMH1 treated cells 
a

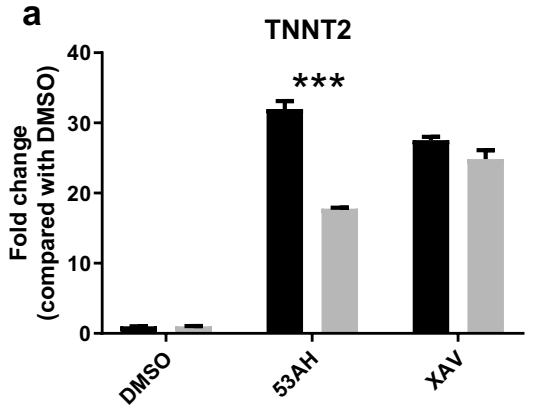

d
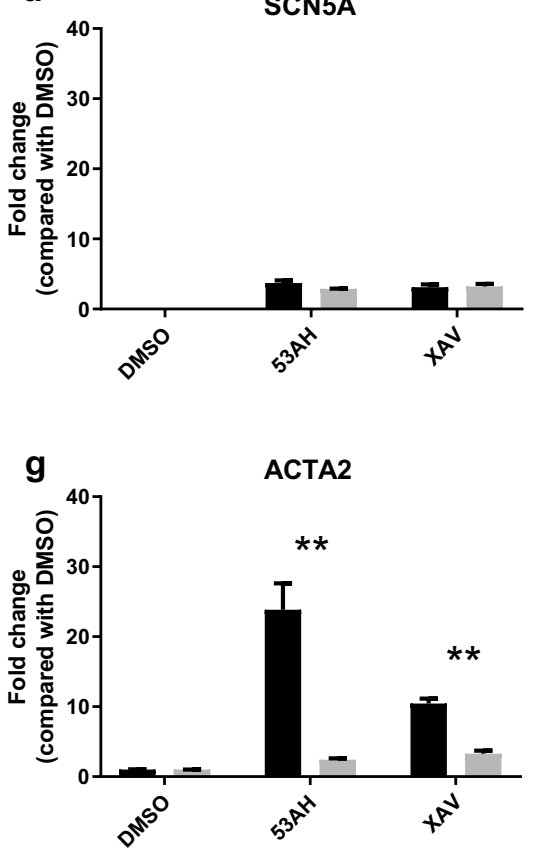

b

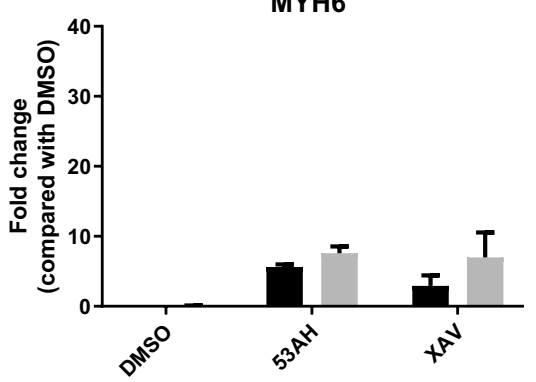

e

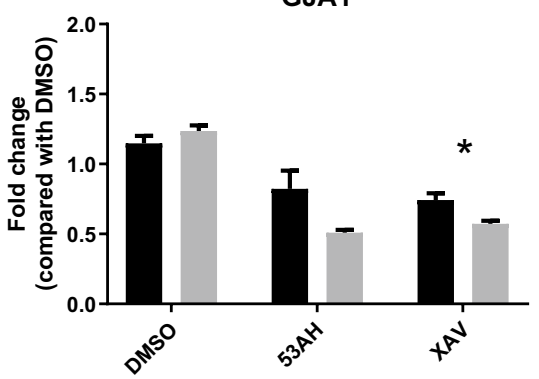

C
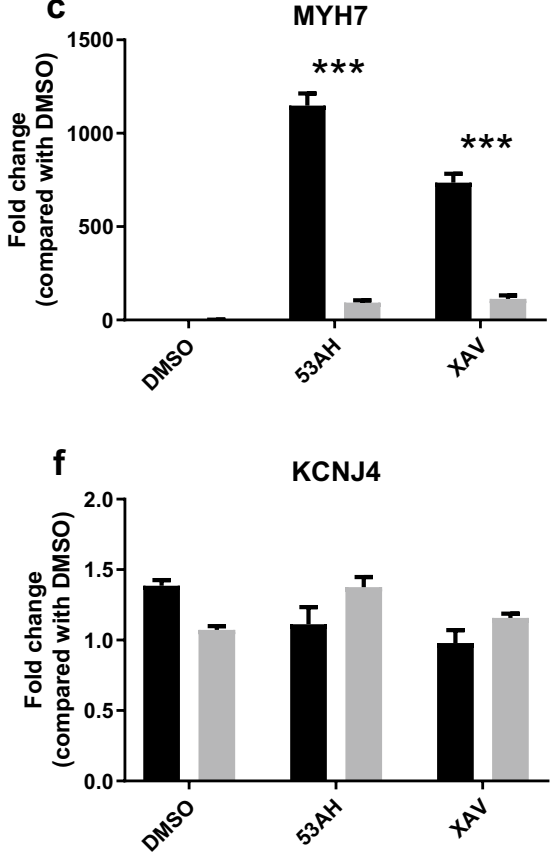

Figure 4. Real time RT-PCR analysis of CPC differentiation treated with Wnt inhibitors in 3D vs 2D culture. Human iPSC-CPCs were plated in 3D aligned nanofiber and 2D plates with the addition of $1.1 \mu \mathrm{M} \mathrm{53AH}$, $10 \mu \mathrm{M}$ XAV939 and DMSO control from day 0 to day 3, followed by culture in assay medium until day 14 of differentiation. The expression of TNNT2, MYH6, MYH7, SCN5A, GJA1, KCNJ4, and ACTA2 were studied, with RPLP0 as a reference control. The relative expression levels of specific genes were calculated according to the formula $2^{-\Delta \mathrm{Ct}}$, and normalized as fold change based on the corresponding DMSO controls. Results are presented as mean \pm SEM. $\mathrm{n}=3 .{ }^{*} \mathrm{p}<0.05,{ }^{* *} \mathrm{p}<0.01,{ }^{* *} \mathrm{p}<0.001$ indicate significant difference between $3 \mathrm{D}$ and $2 \mathrm{D}$ cultures.

in $2 \mathrm{D}$ culture. In the FLIPR assay, DMH1 treated cells in 3D nanofiber plates showed spontaneously synchronized $\mathrm{Ca}^{2+}$ oscillation with an increased oscillation rate in response to isoproterenol at day 14 of differentiation, whereas, only small irregular $\mathrm{Ca}^{2+}$ oscillation was seen in $2 \mathrm{D}$ culture. At day 7, only irregular $\mathrm{Ca}^{2+}$ oscillation was observed in DMH1 treated cells. However, treatment with RepSox or DM induced either no $\mathrm{Ca}^{2+}$ oscillation or only $\mathrm{Ca}^{2+}$ oscillation with very small peak amplitudes in 3D and $2 \mathrm{D}$ cultures at day 7 and 14 .

\section{Discussion}

In vitro cellular models with cells grown in a condition that mimics the in vivo environment have the potential for improving translatability and providing better prediction of the effects of a compound in humans. Aligned nanofiber scaffolds, which structurally mimic the architecture of the cardiac ECM, are attractive model systems for studying cellular phenotypes including cell-cell interactions, proliferation, differentiation and functions. In this study, we investigated the effect of $3 \mathrm{D}$ aligned nanofiber scaffolds on cardiac differentiation of human iPSC-derived CPCs. Our results demonstrated that cells treated with inhibitors of Wnt signaling on 3D aligned nanofiber scaffolds showed: (1) improved CM differentiation of human iPSC-CPCs, as shown with increased number of cTnT-positive cells; (2) elongated nuclear morphology of aSMA-positive cells following the orientation of the aligned nanofibers; (3) reduced compound treatment induced cell proliferation; and (4) synchronized intracellular $\mathrm{Ca}^{2+}$ oscillation in cells. 
a

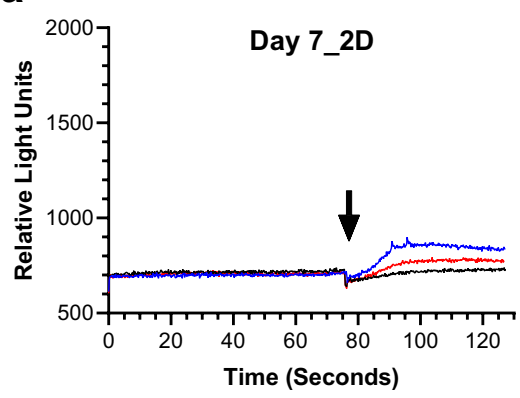

C

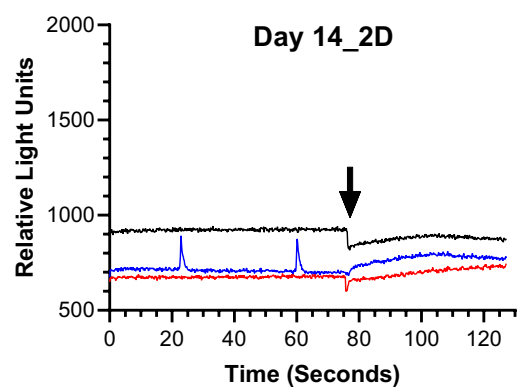

b

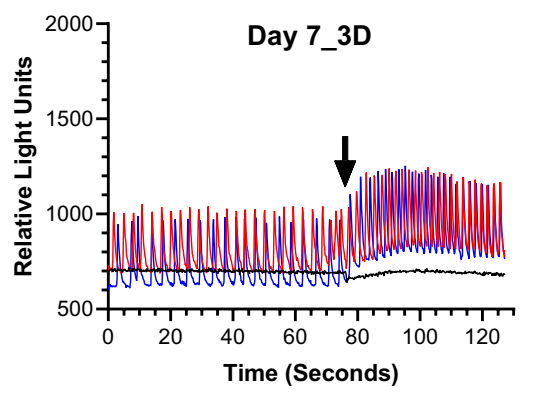

d

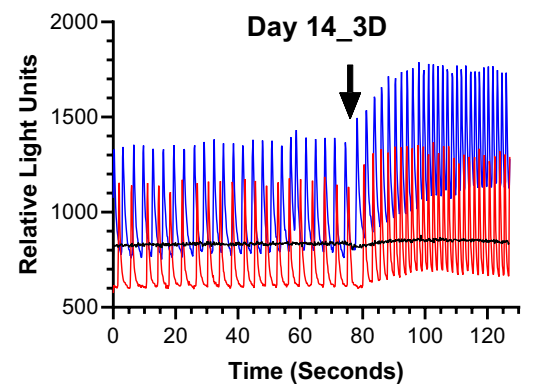

e

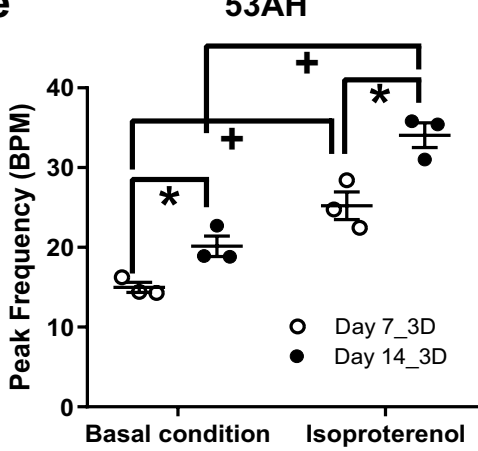

f

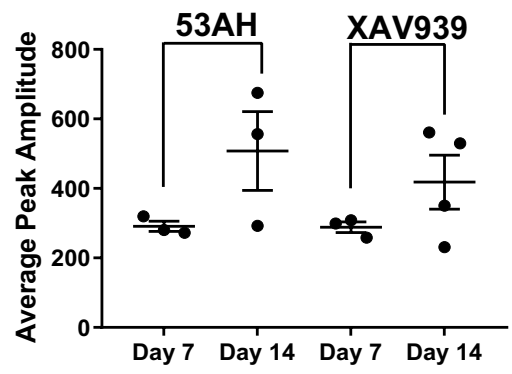

Figure 5. Effect of Wnt signaling inhibition on $\mathrm{Ca}^{2+}$ oscillation of CMs differentiated from CPCs in $3 \mathrm{D}$ vs $2 \mathrm{D}$ culture. Human iPSC-CPCs were plated in 3D aligned nanofiber and 2D plates with the addition of 53AH, XAV939 and DMSO control from day 0 to day 3, followed by culture in assay medium until day 7 or day 14 of differentiation. The cells were then loaded with FLIPR Calcium 5 dye and intracellular $\mathrm{Ca}^{2+}$ oscillation was recorded on a FLIPR Tetra system. (a-d) Representative recordings of intracellular $\mathrm{Ca}^{2+}$ oscillation from cells treated with 1.1 $\mu \mathrm{M}$ 53AH (blue tracers), $10 \mu \mathrm{M}$ XAV939 (red tracers) or DMSO control (black tracers), and cultured in 2D plates for 7 days (a), 14 days (b) and in 3D aligned nanofiber plates for 7 days (c) and 14 days (d) with arrows indicating the time point of adding isoproterenol. (e,f) The peak frequency quantification of $\mathrm{Ca}^{2+}$ oscillation from cells differentiated with $53 \mathrm{AH}$ in $3 \mathrm{D}$ aligned nanofiber culture under the basal and isoproterenol stimulated conditions (e), and the peak amplitude of $\mathrm{Ca}^{2+}$ oscillation in the basal condition in cells treated with $53 \mathrm{AH}$ or XAV939 (f). Quantification results are presented as scatter dot plots with mean \pm SEM. $\mathrm{n}=3-4 .{ }^{*} \mathrm{p}<0.05$ indicates significant difference between Day 7_3D and Day 14_3D cultures. ${ }^{+} \mathrm{p}<0.05$ indicates significant difference between the basal and isoproterenol stimulated conditions.

Treating human iPSC-CPCs with Wnt inhibitors (53AH and XAV939) resulted in a significant increase in $\%$ cTnT-positive cells in 2D culture at day 14 of differentiation compared to cells treated with DMSO vehicle control. These results are consistent with previous reports demonstrating that the Wnt signaling pathway plays important roles in cardiac differentiation ${ }^{10,39,42-45}$.

Advantages of using 3D nanofiber scaffolds to support cell-cell interactions, direct cell alignment, and enhance CM differentiation of stem cells have previously been reported. It has been demonstrated that the expression of cardiac markers (e.g. cTnT and GATA4) were increased in 5-aza-2-deoxycytidine-treated human adipose-derived stem cells seeded on aligned or random nanofiber scaffolds compared to those in 2D cultures ${ }^{37}$. Chen and colleagues reported that $3 \mathrm{D}$ nanofibers combined with activation of Wnt $/ \beta$-catenin signaling during the early period of cell differentiation could synergistically promote the CM differentiation of mouse iPSCs ${ }^{46}$. 3D culture conditions have also been shown to favor CM differentiation of ESC-derived and iPSC-derived cardiovascular progenitors ${ }^{29}$. These reports prompted us to investigate the effect of 3D culture on iPSC-CPC differentiation using 3D aligned nanofiber scaffolds which mimic the architecture of the native ECM of the heart. Our results show that the \% cTnT-positive cells and cTnT intensity of cTnT-positive cells were higher in cells treated with the Wnt inhibitors 53AH or XAV939 on 3D aligned nanofiber scaffolds compared to 2D culture at day 7 or 14 of differentiation, demonstrating an improved CM differentiation in 3D aligned nanofiber culture. This is supported by the RT-PCR analyses showing that the expression levels of the CM marker genes TNNT2 and MYH7 were higher in 53AH treated cells, and MYH7 expression was higher in XAV939 treated cells seeded on 3D aligned nanofiber scaffolds compared with 2D culture. The increases in MYH7 and ACTA2 in the 3D groups may indicate a more ventricular-like phenotype, as MYH7 is predominantly expressed in fetal ventricles whereas MYH6 is found at higher levels in the atrium ${ }^{47,48}$ and ACTA2 expression has been shown to be enriched in ventricular-like cells in vitro ${ }^{49}$. Cell contraction is a characteristic of functional CMs, and synchronized $\mathrm{Ca}^{2+}$ oscillation has been shown to be a surrogate for assessing CM contraction. Furthermore, the FLIPR Ca ${ }^{2+}$ assay results and visual inspection show that the cells treated with the Wnt inhibitors and seeded on 3D aligned nanofiber scaffolds display spontaneous synchronized intracellular $\mathrm{Ca}^{2+}$ oscillation and cell contraction. These differentiated CMs also responded to adrenergic stimulation with increased $\mathrm{Ca}^{2+}$ oscillation frequency. However, 


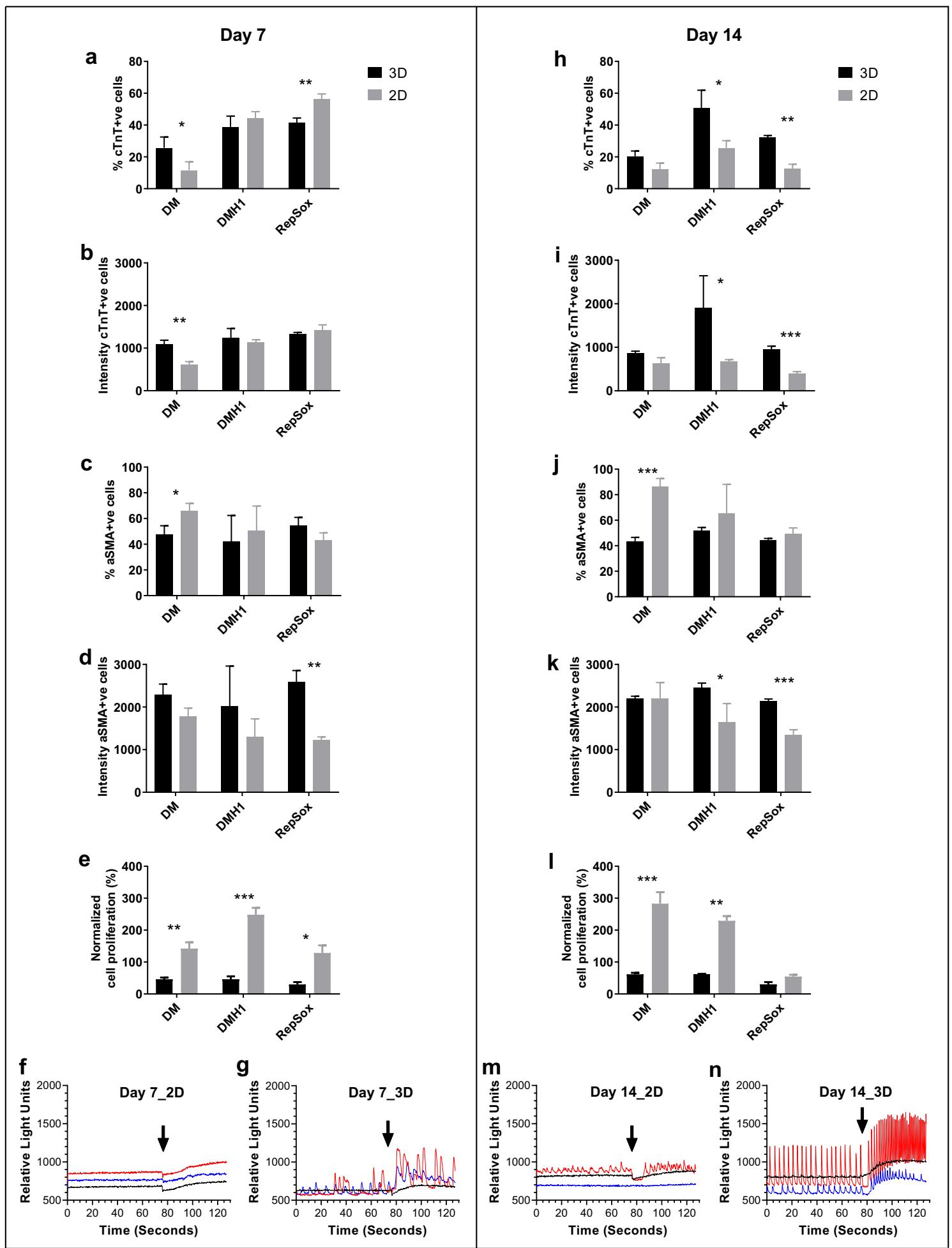

Figure 6. Effect of BMP signaling inhibition and ALK5 inhibitor on CPC differentiation in 3D vs 2D culture. Human iPSC-CPCs were plated in 3D aligned nanofiber and 2D plates with the addition of 1.1 $\mu \mathrm{M}$ DM, $1.1 \mu \mathrm{M}$ DMH1 and $10 \mu \mathrm{M}$ RepSox from day 0 to day 3, followed by culture in assay medium until day 7 or day 14 of differentiation. (a-e) At day 7 of differentiation in 2D and 3D cultures, \% cTnT-positive cells, cTnT intensity of cTnT-positive cells, \% of aSMA-positive cells, aSMA intensity of aSMA-positive cells, and effect on cell proliferation, $(\mathbf{f}-\mathbf{g})$ representative recordings of intracellular $\mathrm{Ca}^{2+}$ oscillation from cells treated with DM (blue tracers), DMH1 (red tracers) or RepSox (black tracers) in 2D and 3D culture for 7 days. Arrows indicate the time point of adding isoproterenol. (h-n) At day 14 of differentiation in 2D and 3D cultures, \% cTnT-positive cells, cTnT intensity of cTnT-positive cells, \% of aSMA-positive cells, aSMA intensity of aSMA-positive cells, effect on cell proliferation and intracellular $\mathrm{Ca}^{2+}$ oscillation. The effect on cell proliferation was normalized as the percentage of cell proliferation based on the on-plate DMSO controls. Results are presented as mean \pm SEM. $\mathrm{n}=3 .{ }^{*} \mathrm{p}<0.05,{ }^{* *} \mathrm{p}<0.01,{ }^{* *} \mathrm{p}<0.001$ indicate significant differences between $3 \mathrm{D}$ and $2 \mathrm{D}$ cultures. 
in $2 \mathrm{D}$ culture, only small irregular or no synchronized $\mathrm{Ca}^{2+}$ oscillations were detected. The $\mathrm{Ca}^{2+}$ oscillation results are consistent with the immunofluorescence data showing a higher percentage and high cTnT intensity of cTnTpositive cells in 3D nanofiber culture treated with $53 \mathrm{AH}$ and XAV939 compared with 2D culture. These results demonstrate that human iPSC-CPCs treated with the inhibitors of Wnt signaling pathway in 3D nanofiber culture were found to be more committed to CM differentiation with synchronized $\mathrm{Ca}^{2+}$ oscillation and cell contraction than cells in $2 \mathrm{D}$ culture, and are in line with previous studies showing that simply manipulating the topography of the surface using 3D nanofiber scaffolds on which cells adhered to is able to improve CM differentiation. 3D aligned nanofiber scaffolds might increase cell-scaffold interactions via increased surface/volume ratio, which may contribute to the improved CM differentiation.

Adult CMs in the heart have an elongated morphology. Our nanofiber validation data demonstrate that human iPSC-CMs, seeded on 3D aligned nanofiber plates, show an elongated morphology that resembles native CMs in the heart, which is consistent with the results reported by several research groups ${ }^{27,28}$. These data suggest that aligned nanotopographic cues can guide CM cell alignment along the direction of fiber orientation and promote adaptation of an elongated CM morphology. Similar effects on cell morphology were reported for SMCs and fibroblastts ${ }^{23,50,51}$. However, CMs differentiated from iPSC-CPCs on 3D aligned nanofibers did not show cell alignment following the orientation of the nanofibers. The images taken at three planes from the confocal microscope showed that the cells located closer to the nanofibers were predisposed towards aSMApositive cell differentiation and cells located further away from the nanofibers were predominately CMs. cTnTpositive CMs seemed not to be attaching to the fibers or penetrate into the spaces between the fibers, whereas the aSMA-positive cells were elongated in alignment with the fibers. The mechanism for the observed differences in aSMA-positive cells and cTnT-positive CMs location and morphology is unclear. Immature CMs and hESCCMs cultured in vitro have been shown to express aSMA protein and ACTA2 gene ${ }^{52,53}$. Therefore, the observed aSMA-positive cells may represent a fraction of immature CMs, which can contribute to the improved $\mathrm{Ca}^{2+}$ oscillation and cell contraction as aSMA-positive CMs can show spontaneous $\mathrm{Ca}^{2+}$ oscillation and cell contraction. It would be interesting to study further if cardiac and smooth muscle cell maturation under the described conditions in $3 \mathrm{D}$ nanofiber culture is also contributing to the improved $\mathrm{Ca}^{2+}$ oscillation.

In a similar manner to our observations with the Wnt inhibitor treatment, the BMP inhibitor DMH1 was very effective at inducing CM differentiation in $3 \mathrm{D}$ culture, as shown with synchronized $\mathrm{Ca}^{2+}$ oscillation, high $\%$ cTnT-positive cells and high cTnT intensity of cTnT-positive cells in 3D culture, but only small/irregular Ca ${ }^{2+}$ oscillations and lower \% cTnT positive cells as well as lower cTnT intensity in 2D culture at day 14. The DMH1 data further support the suggestion that $3 \mathrm{D}$ scaffolds can be a more relevant in vitro model system for studying CPC differentiation and for phenotypic screening of compounds inducing CPC differentiation to CMs as the observed improvement in 3D culture is not only limited for the Wnt signaling pathway inhibition. Another less selective BMP inhibitor DM was not very effective at inducing CM differentiation as it induced only $20 \%$ cTnTpositive cells and small/irregular $\mathrm{Ca}^{2+}$ oscillations in $3 \mathrm{D}$ culture. Furthermore, treatment with the ALK5 inhibitor RepSox did not induce synchronized $\mathrm{Ca}^{2+}$ oscillation either in $3 \mathrm{D}$ or in $2 \mathrm{D}$ culture although there were $41 \%$ and $56 \%$ cTnT positive cells, respectively, in $3 \mathrm{D}$ versus $2 \mathrm{D}$ culture at day 7 . These results suggest that measuring only cardiac marker protein expression may not be enough for the identification of the most effective compounds and may lead to the selection of false positives, and highlight it is important to study and characterize compounds with a combination of marker protein measurement and functional $\mathrm{Ca}^{2+}$ oscillation readout of $\mathrm{CMs}$. In addition, our results suggest the nanofiber scaffolds may play a role in the Wnt and BMP pathway regulation as human iPSC-CPCs treated with the inhibitors of the Wnt or BMP pathways in 3D nanofiber culture were found to be more committed to CM differentiation than cells in 2D culture. However, the mechanism for this is unclear and requires further investigation.

Many reports have indicated that 3D culture can affect cell proliferation. However, the effect of how 3D culture affects cell proliferation, as reported in the literature, is controversial. 3D culture was reported to induce a reduction in cell proliferation for cardiac fibroblasts within alginate scaffolds and for human adipose-derived stem cells on aligned and random PCL nanofiber scaffolds ${ }^{15,54}$. On the contrary, increased cell viability and proliferation was reported for human adipose-derived stem cells on aligned and random PCL nanofibrous scaffolds compared with $2 \mathrm{D}$ culture in a CM differentiation study of human adipose-derived stem cells. Our results are consistent with Dar and Brännmark et al. and showed that human iPSC-CPC proliferation was significantly diminished in the $3 \mathrm{D}$ aligned nanofiber culture together with improved cell differentiation. The theory that cell proliferation is in opposition to differentiation is supported by our results suggesting that CPCs need to be withdrawn from the cell cycle before cardiac differentiation and aligned nanofiber scaffolds may promote cardiac differentiation by switching off CPC proliferation.

In this study, we use a population of CPCs which are clearly defined and well described and characterized. However, a range of populations of CPCs can be obtained from different sources and genetic backgrounds after differentiation. As a next step it would be interesting to utilize the described nanofiber scaffolds and conditions to assess and compare the differentiation characteristics of these different CPC populations. In addition, the iPSCCPCs used in this study is an enriched population of CPCs (characterized with $84.8 \% \pm 3.4 \% \mathrm{KDR}^{\text {pos }} \mathrm{cKIT}^{\text {neg }}$, 83.0\% $\pm 2.7 \% \mathrm{KDR}^{\text {pos }} \mathrm{PDGFR} \alpha^{\text {pos }}$, and $\left.76.5 \% \pm 6.0 \% \mathrm{NKX}^{2.5}{ }^{\text {pos }} \mathrm{CTnT}^{\text {neg }}\right)^{10}$. A useful next step could be to further sort the CPCs of interest from the broader cell population to study the specific phenotype in closer detail. It will also be informative to increase the $3 \mathrm{D}$ nature of the nanofiber scaffolds further to see how far the phenotype can be enhanced. 


\section{Conclusions}

In summary, our study presents evidence that $3 \mathrm{D}$ aligned nanofiber scaffolds, with increased surface/volume ratio and aligned topography of the surface on which cells are adhered, improve iPSC-CPC differentiation to functional CMs across several means of inducing CM differentiation (Wnt and BMP inhibition) as shown with increased cTnT expression and synchronized intracellular $\mathrm{Ca}^{2+}$ oscillation. This work highlights the importance of using a more relevant in vitro model system and measuring not only the expression of CM marker proteins but also the functional readout of CMs in a screen in order to identify the best compounds and to investigate the resulting biology.

\section{Materials and methods}

Materials. Cryopreserved human iCell Cardiac Progenitor Cells (iPSC-CPCs) and human iCell Cardiomyocytes (iPSC-CMs) were purchased from Cellular Dynamics International (Madison, WI, USA) and stored in the vapour phase of liquid nitrogen until use. All chemical reagents (53AH, XAV939, Dorsomorphin (DM), Dorsomorphin homologue 1 (DMH1) and RepSox) were purchased from Sigma-Aldrich Sweden AB (Stockholm, Sweden) or synthesized at AstraZeneca unless stated otherwise. Cell culture medium and supplements were purchased from ThermoFisher Scientific (Stockholm, Sweden). FLIPR Calcium 5 Assay Kit was purchased from Molecular Devices (Berkshire, UK). RNA purification kits were purchased from Qiagen AB (Copenhagen, Denmark). TaqMan gene expression assays were purchased from ThermoFisher Scientific. 384-well and 96-well 2D Corning plates were purchased from VWR (Stockholm, Sweden) and 384-well and 96-well 3D aligned nanofiber plates were purchased from Nanofiber Solutions (Columbus, OH, USA). The thickness of the fiber layers is $\sim 20 \mu \mathrm{m}$ and the nanofiber polymers are approximate $700 \mathrm{~nm}$ in diameter (https://nanofibersolutions.com/).

Cell culture and treatment. Cryopreserved human iPSC-CPCs (CPC-301-020-001-PT, Cellular Dynamics International) were thawed according to the manufacturer's instructions and suspended in CPCs assay medium (William's E medium supplemented with Cell Maintenance Cocktail B) (William's E medium, A1217601, Cell Maintenance Cocktail B, CM4000, ThermoFisher Scientific). Cell number and cell viability were determined on a Cedex counter (Innovatis, Bielefeld, Germany). 2D black clear bottom 384-well plates (3,712/3,764, Corning) and 96-well plates (3,903, Corning), 3D 384-well aligned nanofiber plates (38,402, Nanofiber Solutions) and 96-well aligned nanofiber plates $(9,602$, Nanofiber Solutions) were coated with human fibronectin $(1 \mu \mathrm{g} /$ $\mathrm{ml}$; Roche Applied Science) for at least $2 \mathrm{~h}$ at $37^{\circ} \mathrm{C}$. Compounds XAV939 (X3004, Sigma-Aldrich), 53AH, DM, DMH1 and RepSox were dissolved in dimethyl sulfoxide (DMSO) (D4540, Sigma-Aldrich) as 1,000 $\times$ stock solutions. For the 384-well imaging or FLIPR experiments, compounds were dosed in $50 \mathrm{nl} /$ well using an Echo liquid handler (Labcyte, Sunnyvale, CA, USA) to fibronectin coated plates containing $25 \mu \mathrm{l} /$ well of CPCs assay medium. DMSO was used as the vehicle control with a $0.1 \%$ final concentration. To compensate for the large surface area of 3D nanofiber plate compared to conventional 2D plates, a higher cell seeding density was initially used for the nanofiber plates. iPSC-CPCs were seeded to plates containing pre-dosed compounds at a density of 13,000 cells/well in 2D plates and 17,000 cells/well in 3D aligned nanofiber plates. For the 96 -well gene expression experiments, compounds were dosed to fibronectin coated plates by adding $40 \mu \mathrm{l} / \mathrm{well}$ of $1 / 200$ pre-diluted DMSO or compounds. iPSC-CPCs were seeded at 40,000 cells/well in 2D plates or 52,000 cells/well in 3D plates in $160 \mu \mathrm{l}$ of CPCs assay medium. Cells with the addition of compounds from day 0 to day 3 were incubated at $37^{\circ} \mathrm{C}$ with $5 \% \mathrm{CO}_{2}$, followed by culture in fresh assay medium with medium refreshment every 48 or $72 \mathrm{~h}$ until the experiments were terminated at day 7 or day 14.

Cryopreserved human iPSC-CMs (CMC-100-110-001, Cellular Dynamics International) were thawed and suspended in iCell CM plating medium (CMM-100-110-005, Cellular Dynamics International) as described previously ${ }^{55}$. Cell number and viability were determined on a Cedex counter (Innovatis) and total cell number was determined with cell plating efficiency taken into account. Cells were seeded in $50 \mu \mathrm{l} /$ well at 4,000 cells/ well in gelatin-coated 384-well 2D Corning black clear bottom plates and at 6,000 cells/well in gelatin coated 384-well 3D aligned nanofiber plates and incubated at $37^{\circ} \mathrm{C}$ for $48 \mathrm{~h}$. The medium was then changed to iCell CM maintenance medium (CMM-100-120-005, Cellular Dynamics International) and maintained at $37^{\circ} \mathrm{C}$ for 10 days with medium refreshment every 48 or $72 \mathrm{~h}$.

Immunofluorescence. Cells were fixed in $4 \%(\mathrm{w} / \mathrm{v})$ formaldehyde (dilute $37 \%$ formaldehyde solution with PBS, F8775, Sigma-Aldrich) on the indicated day for $20 \mathrm{~min}$ at room temperature (RT), permeabilized with $0.1 \%$ Triton X-100 in PBS for 15 min and then blocked in 5\% FBS/TPBS block buffer (with 0.02\% Tween-20 in PBS) for $30 \mathrm{~min}$. The cells from the CPCs differentiation study were then stained with primary antibodies [rabbit antiCardiac Troponin T (cTnT) (1:400, ab45932, Abcam), mouse anti-human smooth muscle actin (aSMA) (1:100, Clone 1A4, M0851, Dako)] overnight at $4^{\circ} \mathrm{C}$. After overnight incubation, cells were washed with PBS followed by 45 min incubation with secondary antibodies Alexa Fluor 647 Donkey anti-rabbit IgG $(\mathrm{H}+\mathrm{L})(1: 500, \mathrm{~A}-31573$, ThermoFisher Scientific), Alexa Fluor 488 Goat anti-mouse IgG (H+L) (1:500, A-11001, ThermoFisher Scientific) and 20 min with Hoechst 33342 (62249, ThermoFisher Scientific) at RT followed by final washing with PBS. The iPSC-CMs were stained with primary antibody rabbit anti-cTnT followed by secondary antibody Alexa Fluor 488 Goat anti-rabbit IgG (H+L) (1:500, A-11034, ThermoFisher Scientific) and Hoechst 33342. The plates were sealed and imaged on an ImageXpress XL system (Molecular Devices, Berkshire, UK) using $20 \times(4$ sites per well) or $4 \times$ (one site per well) objectives. The plates with 14 -day differentiation were also imaged on a Yokogawa CV7000 confocal microscope (Wako automation, San Diego, California, USA) using a $20 \times$ air objective and $6 \mu \mathrm{m}$ intervals. Images were analyzed using Columbus image analysis software (PerkinElmer). 
Real-time reverse transcription polymerase chain reaction (RT-PCR). For analysis of the gene expression, treated and untreated cells were washed with PBS and lysed using RNeasy cell lysis buffer RLT (RNeasy Mini kit, 74104, Qiagen, Copenhagen, Denmark) to obtain $350 \mu$ l cell lysate/sample. The total RNA was purified with RNeasy Mini kit using RNA Cell Method with DNase digest protocol and $30 \mu$ l elution volume on a robotic RNA purification system QIAcube (Qiagen) following the instructions from the manufacturer. Purified RNA samples were stored at $-80^{\circ} \mathrm{C}$ until analysis. mRNA was reverse transcribed to CDNA using random primers and High capacity cDNA reverse transcription kit (4368813, Applied Biosystems, Foster City, CA, USA). Real time RT-PCR was conducted with Taqman technology to assess gene expression of the selected human genes, TNNT2 (cardiac troponin T, Hs00165960_m1, ThermoFisher Scientific), MYH6 (myosin heavy chain 6, Hs01101425_m1, ThermoFisher Scientific), MYH7 (myosin heavy chain beta, Hs01110632_m1, ThermoFisher Scientific), SCN5A (Nav1.5, Hs00165693_m1, ThermoFisher Scientific), KCNJ4 (inward rectifier potassium channel 4, Hs00705379_s1, ThermoFisher Scientific), GJA1 (gap junction protein alpha 1, Hs00748445_s1, ThermoFisher Scientific), and ACTA2 (alpha smooth muscle actin, Hs00426835_g1, ThermoFisher Scientific). Ribosomal Protein Large P0 (RPLP0), using forward primer 5'-3' CCATTCTATCATCAACGGGTACA, reverse primer 5'-3' AGCAAGTGGGAAGGTGTAATCC and probe 5' FAM-TCTCCACAGACAAGGCCAGGACTC GT-TAMRA 3' (Sigma-Aldrich), was used as endogenous control. Fourty cycles of reactions were performed in triplicates for each sample on an Applied Biosystems QuantStudio 7 instrument (ThermoFisher Scientific). Only samples reaching threshold values before cycle 36 were included. The relative expression levels of specific genes were calculated using the formula $2^{-\mathrm{dCt}}$, where $\mathrm{dCt}$ is the difference in threshold cycle $(\mathrm{Ct})$ values between the target gene and the endogenous control gene RPLP0.

Intracellular calcium oscillation. At day 7 or day 14 of iPSC-CPCs differentiation, or at day 10 of iPSCCMs maintenance, intracellular $\mathrm{Ca}^{2+}$ oscillation was studied on a FLIPR Tetra system (Molecular Devices, Berkshire, UK) using FLIPR Calcium 5 Assay Kit (Molecular Devices) as described by Pointon et al. ${ }^{55}$. Briefly, the cell culture media were first removed and replaced with $25 \mu \mathrm{l} /$ well of fresh culture medium $2 \mathrm{~h}$ prior to assay. The cells were loaded with $1 \times$ FLIPR Calcium 5 dye at $37^{\circ} \mathrm{C}$ for $40 \mathrm{~min}$ by adding $25 \mu \mathrm{l} /$ well of $2 \times$ FLIPR Calcium 5 dye. The cell plates were transferred to a FLIPR Tetra system and maintained at $37^{\circ} \mathrm{C}^{-\mathrm{Ca}^{2+} \text { oscillations }}$ were recorded at Ex $485 \mathrm{~nm} / \mathrm{Em} 530 \mathrm{~nm}$ with $0.05 \mathrm{~s}$ exposure time/read and $0.12 \mathrm{~s}$ read interval. $450 \mathrm{reads}$ were collected at the basal condition, followed by a further 350 reads post-the addition of $12.5 \mu \mathrm{l} /$ well of $10 \mu \mathrm{M}$ Isoproterenol solution (prepared from $10 \mathrm{mM}$ stock, I5627, Sigma-Aldrich, diluted in assay medium) to reach a final concentration of $2 \mu \mathrm{M}$ isoproterenol using onboard liquid handling within the FLIPR Tetra, allowing both pre- and post- compound reads to be obtained from the same well from plates maintained at $37^{\circ} \mathrm{C}$ during the recording period. $\mathrm{Ca}^{2+}$ oscillation peak frequencies and peak amplitude were analyzed using the ScreenWorks Peak Pro Software (Molecular Devices).

Statistical analysis. Data are presented as mean \pm SEM $(n=3)$. Statistical analysis was performed with multiple $t$ tests correct for multiple comparisons using the Holm-Sidak method in GraphPad Prism 7 (GraphPad Software, California, USA). Value of $\mathrm{p}<0.05$ was considered to be significant and was indicated with ${ }^{\star} \mathrm{p}<0.05$, ${ }^{* *} \mathrm{p}<0.01$ and ${ }^{* * *} \mathrm{p}<0.001$

Received: 3 March 2020; Accepted: 30 July 2020

Published online: 11 August 2020

\section{References}

1. Lloyd-Jones, D. et al. Heart disease and stroke statistics-2009 update: a report from the American Heart Association Statistics Committee and Stroke Statistics Subcommittee. Circulation 119, e21-181. https://doi.org/10.1161/CIRCULATIONAHA.108.19126 1 (2009).

2. Laflamme, M. A. \& Murry, C. E. Heart regeneration. Nature 473, 326-335. https://doi.org/10.1038/nature10147 (2011).

3. Breckwoldt, K., Weinberger, F. \& Eschenhagen, T. Heart regeneration. Biochim. Biophys. Acta 1863, 1749-1759. https://doi. org/10.1016/j.bbamcr.2015.11.010 (2016)

4. Plowright, A. T., Engkvist, O., Gill, A., Knerr, L. \& Wang, Q. D. Heart regeneration: opportunities and challenges for drug discovery with novel chemical and therapeutic methods or agents. Angew. Chem. Int. Ed .Engl. 53, 4056-4075. https://doi.org/10.1002/ anie.201307034 (2014).

5. Foo, K. S. et al. Human ISL1(+) ventricular progenitors self-assemble into an in vivo functional heart patch and preserve cardiac function post infarction. Mol. Ther. 26, 1644-1659. https://doi.org/10.1016/j.ymthe.2018.02.012 (2018).

6. Liu, Y. W. et al. Human embryonic stem cell-derived cardiomyocytes restore function in infarcted hearts of non-human primates. Nat. Biotechnol. 36, 597-605. https://doi.org/10.1038/nbt.4162 (2018).

7. Miyagawa, S. \& Sawa, Y. Building a new strategy for treating heart failure using induced pluripotent stem cells. J. Cardiol. 72, 445-448. https://doi.org/10.1016/j.jjcc.2018.05.002 (2018).

8. Gao, L. et al. Large cardiac muscle patches engineered from human induced-pluripotent stem cell-derived cardiac cells improve recovery from myocardial infarction in swine. Circulation 137, 1712-1730. https://doi.org/10.1161/CIRCULATIONAHA.117.03078 5 (2018).

9. Menasche, P. et al. Transplantation of human embryonic stem cell-derived cardiovascular progenitors for severe ischemic left ventricular dysfunction. J. Am. Coll. Cardiol. 71, 429-438. https://doi.org/10.1016/j.jacc.2017.11.047 (2018).

10. Drowley, L. et al. Human induced pluripotent stem cell-derived cardiac progenitor cells in phenotypic screening: a transforming growth factor-beta type 1 receptor kinase inhibitor induces efficient cardiac differentiation. Stem Cells Transl. Med. 5, 164-174. https://doi.org/10.5966/sctm.2015-0114 (2016).

11. Yamada, K. M. \& Cukierman, E. Modeling tissue morphogenesis and cancer in 3D. Cell 130, 601-610. https://doi.org/10.1016/j. cell.2007.08.006 (2007). 
12. Guilak, F. et al. Control of stem cell fate by physical interactions with the extracellular matrix. Cell Stem Cell 5, 17-26. https://doi. org/10.1016/j.stem.2009.06.016 (2009).

13. Hwang, N. S., Varghese, S. \& Elisseeff, J. Cartilage tissue engineering: directed differentiation of embryonic stem cells in threedimensional hydrogel culture. Methods Mol. Biol. 407, 351-373. https://doi.org/10.1007/978-1-59745-536-7_24 (2007).

14. Bell, A., Kofron, M. \& Nistor, V. Multiphoton crosslinking for biocompatible 3D printing of type I collagen. Biofabrication 7, 035007. https://doi.org/10.1088/1758-5090/7/3/035007 (2015).

15. Brannmark, C. et al. Increased adipogenesis of human adipose-derived stem cells on polycaprolactone fiber matrices. PLoS ONE 9, e113620. https://doi.org/10.1371/journal.pone.0113620 (2014).

16. Christopherson, G. T., Song, H. \& Mao, H. Q. The influence of fiber diameter of electrospun substrates on neural stem cell differentiation and proliferation. Biomaterials 30, 556-564. https://doi.org/10.1016/j.biomaterials.2008.10.004 (2009).

17. Bellis, A. D. et al. Dynamic transcription factor activity profiling in 2D and 3D cell cultures. Biotechnol. Bioeng. 110, 563-572. https://doi.org/10.1002/bit.24718 (2013).

18. Alemany-Ribes, M., Garcia-Diaz, M., Busom, M., Nonell, S. \& Semino, C. E. Toward a 3D cellular model for studying in vitro the outcome of photodynamic treatments: accounting for the effects of tissue complexity. Tissue Eng. Part A 19, 1665-1674. https:// doi.org/10.1089/ten.TEA.2012.0661 (2013).

19. Breslin, S. \& O'Driscoll, L. Three-dimensional cell culture: the missing link in drug discovery. Drug Discov. Today 18, 240-249. https://doi.org/10.1016/j.drudis.2012.10.003 (2013).

20. Kelm, J. M., Timmins, N. E., Brown, C. J., Fussenegger, M. \& Nielsen, L. K. Method for generation of homogeneous multicellular tumor spheroids applicable to a wide variety of cell types. Biotechnol. Bioeng. 83, 173-180. https://doi.org/10.1002/bit.10655 (2003).

21. Cukierman, E., Pankov, R. \& Yamada, K. M. Cell interactions with three-dimensional matrices. Curr. Opin. Cell Biol. 14, 633-639 (2002).

22. Kim, D., Kim, S. M., Lee, S. \& Yoon, M. H. Investigation of neuronal pathfinding and construction of artificial neuronal networks on 3D-arranged porous fibrillar scaffolds with controlled geometry. Sci. Rep. 7, 7716. https://doi.org/10.1038/s41598-017-08231 -3 (2017).

23. Yim, E. K. et al. Nanopattern-induced changes in morphology and motility of smooth muscle cells. Biomaterials 26, 5405-5413. https://doi.org/10.1016/j.biomaterials.2005.01.058 (2005).

24. Hurrell, T., Lilley, K. S. \& Cromarty, A. D. Proteomic responses of HepG2 cell monolayers and 3D spheroids to selected hepatotoxins. Toxicol. Lett. 300, 40-50. https://doi.org/10.1016/j.toxlet.2018.10.030 (2019).

25. Chitrangi, S., Nair, P. \& Khanna, A. Three-dimensional polymer scaffolds for enhanced differentiation of human mesenchymal stem cells to hepatocyte-like cells: a comparative study. J. Tissue Eng. Regen. Med. 11, 2359-2372. https://doi.org/10.1002/term.2136 (2017).

26. Lemoine, M. D. et al. Human iPSC-derived cardiomyocytes cultured in 3D engineered heart tissue show physiological upstroke velocity and sodium current density. Sci. Rep. 7, 5464. https://doi.org/10.1038/s41598-017-05600-w (2017).

27. Kai, D., Prabhakaran, M. P., Jin, G. \& Ramakrishna, S. Guided orientation of cardiomyocytes on electrospun aligned nanofibers for cardiac tissue engineering. J. Biomed. Mater. Res. B Appl. Biomater. 98, 379-386. https://doi.org/10.1002/jbm.b.31862 (2011).

28. Khan, M. et al. Evaluation of changes in morphology and function of human induced pluripotent stem cell derived cardiomyocytes (HiPSC-CMs) cultured on an aligned-nanofiber cardiac patch. PLoS ONE 10, e0126338. https://doi.org/10.1371/journal.pone.01263 38 (2015).

29. Ruan, J. L. et al. Mechanical stress conditioning and electrical stimulation promote contractility and force maturation of induced pluripotent stem cell-derived human cardiac tissue. Circulation 134, 1557-1567. https://doi.org/10.1161/CIRCULATIO NAHA.114.014998 (2016).

30. Orlova, Y., Magome, N., Liu, L., Chen, Y. \& Agladze, K. Electrospun nanofibers as a tool for architecture control in engineered cardiac tissue. Biomaterials 32, 5615-5624. https://doi.org/10.1016/j.biomaterials.2011.04.042 (2011).

31. Kharaziha, M. et al. Tough and flexible CNT-polymeric hybrid scaffolds for engineering cardiac constructs. Biomaterials 35, 7346-7354. https://doi.org/10.1016/j.biomaterials.2014.05.014 (2014).

32. Li, J. et al. Human pluripotent stem cell-derived cardiac tissue-like constructs for repairing the infarcted myocardium. Stem Cell Rep. 9, 1546-1559. https://doi.org/10.1016/j.stemcr.2017.09.007 (2017).

33. Kang, B. J. et al. Umbilical-cord-blood-derived mesenchymal stem cells seeded onto fibronectin-immobilized polycaprolactone nanofiber improve cardiac function. Acta Biomater. 10, 3007-3017. https://doi.org/10.1016/j.actbio.2014.03.013 (2014).

34. Kang, P. L. et al. Nano-sized collagen I molecules enhanced the differentiation of rat mesenchymal stem cells into cardiomyocytes. J. Biomed. Mater. Res. A 101, 2808-2816. https://doi.org/10.1002/jbm.a.34589 (2013).

35. Jha, R. et al. Simulated microgravity and 3D culture enhance induction, viability, proliferation and differentiation of cardiac progenitors from human pluripotent stem cells. Sci. Rep. 6, 30956. https://doi.org/10.1038/srep30956 (2016).

36. Guan, J. et al. The stimulation of the cardiac differentiation of mesenchymal stem cells in tissue constructs that mimic myocardium structure and biomechanics. Biomaterials 32, 5568-5580. https://doi.org/10.1016/j.biomaterials.2011.04.038 (2011).

37. Safaeijavan, R., Soleimani, M., Divsalar, A., Eidi, A. \& Ardeshirylajimi, A. Comparison of random and aligned PCL nanofibrous electrospun scaffolds on cardiomyocyte differentiation of human adipose-derived stem cells. Iran. J. Basic Med. Sci. 17, 903-911 (2014).

38. Tobita, K., Garrison, J. B., Liu, L. J., Tinney, J. P. \& Keller, B. B. Three-dimensional myofiber architecture of the embryonic left ventricle during normal development and altered mechanical loads. Anat. Rec. A Discov. Mol. Cell Evol. Biol. 283, 193-201. https ://doi.org/10.1002/ar.a.20133 (2005).

39. Ao, A., Hao, J., Hopkins, C. R. \& Hong, C. C. DMH1, a novel BMP small molecule inhibitor, increases cardiomyocyte progenitors and promotes cardiac differentiation in mouse embryonic stem cells. PLoS ONE 7, e41627. https://doi.org/10.1371/journ al.pone.0041627 (2012).

40. Versaevel, M., Grevesse, T. \& Gabriele, S. Spatial coordination between cell and nuclear shape within micropatterned endothelial cells. Nat. Commun. 3, 671. https://doi.org/10.1038/ncomms1668 (2012).

41. Chen, B., Co, C. \& Ho, C. C. Cell shape dependent regulation of nuclear morphology. Biomaterials 67, 129-136. https://doi. org/10.1016/j.biomaterials.2015.07.017 (2015).

42. Wang, H., Hao, J. \& Hong, C. C. Cardiac induction of embryonic stem cells by a small molecule inhibitor of Wnt/beta-catenin signaling. ACS Chem. Biol. 6, 192-197. https://doi.org/10.1021/cb100323z (2011).

43. Lian, X. et al. Directed cardiomyocyte differentiation from human pluripotent stem cells by modulating Wnt/beta-catenin signaling under fully defined conditions. Nat. Protoc. 8, 162-175. https://doi.org/10.1038/nprot.2012.150 (2013).

44. Willems, E. et al. Small-molecule inhibitors of the Wnt pathway potently promote cardiomyocytes from human embryonic stem cell-derived mesoderm. Circ. Res. 109, 360-364. https://doi.org/10.1161/CIRCRESAHA.111.249540 (2011).

45. Patsch, C. et al. Generation of vascular endothelial and smooth muscle cells from human pluripotent stem cells. Nat. Cell Biol. 17, 994-1003. https://doi.org/10.1038/ncb3205 (2015).

46. Chen, Y. et al. Three-dimensional poly-(epsilon-caprolactone) nanofibrous scaffolds directly promote the cardiomyocyte differentiation of murine-induced pluripotent stem cells through Wnt/beta-catenin signaling. BMC Cell Biol. 16, 22. https://doi.org/10.1186/ s12860-015-0067-3 (2015). 
47. Reiser, P. J., Portman, M. A., Ning, X. H. \& SchomischMoravec, C. Human cardiac myosin heavy chain isoforms in fetal and failing adult atria and ventricles. Am. J. Physiol. Heart Circ. Physiol. 280, H1814-H1820. https://doi.org/10.1152/ajpheart.2001.280.4.H1814 (2001).

48. Shadrin, I. Y. et al. Cardiopatch platform enables maturation and scale-up of human pluripotent stem cell-derived engineered heart tissues. Nat. Commun. 8, 1825. https://doi.org/10.1038/s41467-017-01946-x (2017).

49. Potta, S. P. et al. Isolation and functional characterization of alpha-smooth muscle actin expressing cardiomyocytes from embryonic stem cells. Cell Physiol. Biochem. 25, 595-604. https://doi.org/10.1159/000315078 (2010).

50. Agrawal, A. et al. Smooth muscle cell alignment and phenotype control by melt spun polycaprolactone fibers for seeding of tissue engineered blood vessels. Int. J. Biomater. 2015, 434876. https://doi.org/10.1155/2015/434876 (2015).

51. Jia, L., Prabhakaran, M. P., Qin, X. \& Ramakrishna, S. Guiding the orientation of smooth muscle cells on random and aligned polyurethane/collagen nanofibers. J. Biomater. Appl. 29, 364-377. https://doi.org/10.1177/0885328214529002 (2014).

52. Potta, S. P. et al. Functional characterization and gene expression profiling of alpha-smooth muscle actin expressing cardiomyocytes derived from murine induced pluripotent stem cells. Stem Cell Rev. Rep. 8, 229-242. https://doi.org/10.1007/s12015-011-9271-5 (2012).

53. van Laake, L. W. et al. Human embryonic stem cell-derived cardiomyocytes survive and mature in the mouse heart and transiently improve function after myocardial infarction. Stem Cell Res. 1, 9-24. https://doi.org/10.1016/j.scr.2007.06.001 (2007).

54. Dar, A., Shachar, M., Leor, J. \& Cohen, S. Optimization of cardiac cell seeding and distribution in 3D porous alginate scaffolds. Biotechnol. Bioeng. 80, 305-312. https://doi.org/10.1002/bit.10372 (2002).

55. Pointon, A. et al. Assessment of cardiomyocyte contraction in human-induced pluripotent stem cell-derived cardiomyocytes. Toxicol. Sci. 144, 227-237. https://doi.org/10.1093/toxsci/kfu31 (2015).

\section{Acknowledgements}

The authors would like to thank Gabriella Brolén and Anna Forslöw for valuable discussions, Alexander Kvist for supporting the experiments.

\section{Author contributions}

M.D., H.A., S.M., A.S., A,J., Q.-D.W., A.T.P. and L.D. contributed to preparation of the manuscript. M.D., H.A., S.M. and A.S. contributed to acquisition of data. M.D., H.A., S.M. and A.S. contributed to data analysis. M.D., A.T.P. and L.D. contributed to study conception and design.

\section{Competing interests}

The authors declare no competing interests.

\section{Additional information}

Supplementary information is available for this paper at https://doi.org/10.1038/s41598-020-70547-4.

Correspondence and requests for materials should be addressed to M.D.

Reprints and permissions information is available at www.nature.com/reprints.

Publisher's note Springer Nature remains neutral with regard to jurisdictional claims in published maps and institutional affiliations.

Open Access This article is licensed under a Creative Commons Attribution 4.0 International License, which permits use, sharing, adaptation, distribution and reproduction in any medium or format, as long as you give appropriate credit to the original author(s) and the source, provide a link to the Creative Commons license, and indicate if changes were made. The images or other third party material in this article are included in the article's Creative Commons license, unless indicated otherwise in a credit line to the material. If material is not included in the article's Creative Commons license and your intended use is not permitted by statutory regulation or exceeds the permitted use, you will need to obtain permission directly from the copyright holder. To view a copy of this license, visit http://creativecommons.org/licenses/by/4.0/.

(C) The Author(s) 2020 